\title{
Methodologies for Adaptive Flight Envelope Estimation and Protection
}

\author{
Liang Tang ${ }^{1}$, Michael Roemer, Jianhua Ge \\ Impact Technologies, LLC. Rochester, NY 14623 \\ Agamemnon Crassidis \\ Rochester Institute of Technology, Rochester, NY 14623 \\ J.V.R. Prasad, \\ Georgia Institute of Technology, Atlanta, GA 30332 \\ and \\ Christine Belcastro \\ NASA Langley Research Center, Hampton, VA 23681
}

\begin{abstract}
This paper reports the latest development of several techniques for adaptive flight envelope estimation and protection system for aircraft under damage upset conditions. Through the integration of advanced fault detection algorithms, real-time system identification of the damage/faulted aircraft and flight envelop estimation, real-time decision support can be executed autonomously for improving damage tolerance and flight recoverability. Particularly, a bank of adaptive nonlinear fault detection and isolation estimators were developed for flight control actuator faults; a real-time system identification method was developed for assessing the dynamics and performance limitation of impaired aircraft; online learning neural networks were used to approximate selected aircraft dynamics which were then inverted to estimate command margins. As off-line training of network weights is not required, the method has the advantage of adapting to varying flight conditions and different vehicle configurations. The key benefit of the envelope estimation and protection system is that it allows the aircraft to fly close to its limit boundary by constantly updating the controller command limits during flight. The developed techniques were demonstrated on NASA's Generic Transport Model (GTM) simulation environments with simulated actuator faults. Simulation results and remarks on future work are presented.
\end{abstract}

\section{Introduction}

ignificant research has been conducted in recent years on the development and implementation of the Integrated Resilient Aircraft Control (IRAC) technologies for both civil and military aircraft. To improve post-failure flight safety, it is highly desirable to characterize the adverse conditions (faults, damage, and/or upset), and estimate the achievable flight envelope of the aircraft. With the term aerodynamic envelope we refer to the set of states in which control actions exist to ensure that the vehicle can proceed to a safe landing from the air, while constraints on flight path, landing point and velocity are satisfied. The different types of anomalies including failed actuators, failed engine, and damaged surfaces may result in the changes of the aerodynamics coefficients, therefore the real-time estimation of system health, especially the identification of the aerodynamic coefficients and the extrapolation of the identified system dynamics are key steps for modeling the damaged aircraft.

To address this challenging problem, a variety of techniques have been developed in the past few years by researchers from the industry, academia and government. Particularly, researchers at NASA have conducted studies on the aerodynamic effects and modeling of damage to transport aircraft ${ }^{1}$, general equation of motion for damaged

\footnotetext{
${ }^{1}$ Team Lead, Intelligent Control and Prediction, Impact Technologies, LLC. Liang.Tang@impact-tek.com
} 
asymmetric aircraft ${ }^{2}$, and flight dynamics to account for changes in aerodynamics, mass properties, and the center of gravity that can compromise the stability of the damaged aircraft ${ }^{3}$. A recent simulation study was also conducted to investigate aerodynamic modeling methods for prediction of post-stall flight dynamics of large transport airplanes ${ }^{4}$. Evaluation of flight envelope has been tackled with various offline and online methods. Offline methods include wind tunnel testing and high-fidelity model-based computation of attainable equilibrium sets ${ }^{5}$. Online methods include formulating flight envelope estimation as a reachability problem and solved with level set method ${ }^{6,7}$, and Boeing's approach using Control-Centric Modeling, dynamic flexible structure and load models ${ }^{8}$. Formal robustness analysis method has also been used for determining a reliable flight regime in the flight envelope within which an integrated resilient control system can achieve the desired performance in the presence of parameter uncertainty and unmodeled dynamics'.

In conjunction with fault tolerant control aspect, Lombaerts et al developed a nonlinear reconfigurable flight control based on online physical model identification, where the damaged aircraft model is identified and applied in real time to the model-based adaptive nonlinear dynamic inversion routine which allows flight control reconfigurations online ${ }^{10}$. A coauthor of this paper has conducted automatic flight envelope protection technology for autonomous unmanned aerial vehicles where online-learning adaptive neural networks are utilized to generate online dynamic models, which are used to estimate limits on controller commands ${ }^{11}$. In conjunction with adaptive trajectory generation and path planning, Tang et al developed an adaptive flight planner to build landing trajectories for disabled aircraft ${ }^{12}$. A case study was investigated in which a Generalized Transport Model aircraft must land following the loss of a significant fraction of its left wing. In their work, post-damage flight envelope was defined by a set of trimmed flight conditions.

This paper reports the latest development of several novel techniques for adaptive flight envelope estimation and protection. Through the integration of advanced fault detection algorithms, real-time system identification and flight envelop estimation, real-time decision support can be executed autonomously to improve damage tolerance and flight recoverability. Our approach is different from others in many aspects. First, we investigated not only the online system identification of the changes of aerodynamic coefficients, but also the extrapolation/prediction of these changes to other operating conditions. Secondly, we use separated approaches for flight control actuator faults and airframe faults so that the higher technological readiness level (TRL) solutions for actuators faults can be utilized in addition to the general system ID approach. Last but not lease, we highlight the seamless integration of online flight envelope estimation and protection functions as a total solution.

The novelty in this approach is the integration of online system identification (ID), parameter extrapolation and efficient reachable set calculation to estimate and protect the achievable flight envelope after the occurrence of faults. The highlights of the innovation are illustrated in Figure 1 and listed as follows:

- Changes of aerodynamic coefficients are identified by a recursive system ID technique using onboard sensor measurements;

- Predicting the flight envelope at a different flight condition based on the estimation at current and past conditions is addressed by an computationally efficient linear extrapolation method;

- The flight envelope estimation is continuously updated and improved as more flight conditions are visited;

- The computation of the aerodynamic envelope is formulated as a reachability problem and solved efficiently using the level set methods.

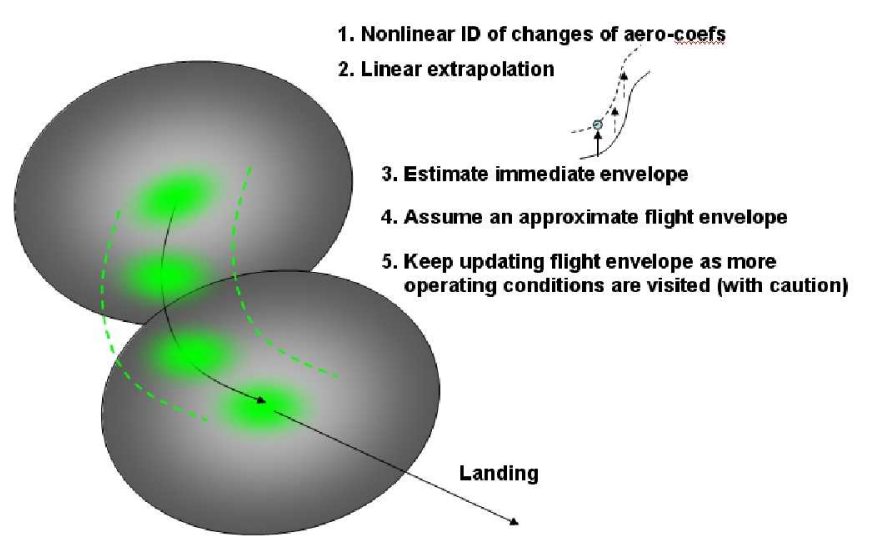

Figure 1. Online System Identification, Parameter Extrapolation \& Progressive Envelope Estimation

- For envelope protection, online learning neural networks are used to approximate selected aircraft dynamics which are then inverted to estimate command margins for limit avoidance.

The purpose of this paper is to provide a brief overview of several methodologies recently developed by the coauthors in a NASA sponsored program. The rest of this paper is organized as follows. Section II gives an overview of the developed system architecture. Section III through Section VI presents a few select methodologies 
that address the key functionalities of the system. Simulation results are presented in Section VII. The last section concludes the paper with remarks on future research directions.

\section{System Overview}

The system architecture for the onboard flight envelope estimation and protection (EEP) system is shown in Figure 2. Sensor measurement signals and state estimates from a state estimator (usually a Kalman or extended Kalman filter) are sent to an anomaly detection module. If the fault is classified as flight control actuator fault, diagnostic routines that are more appropriate for the problem ${ }^{13,14,15}$ will be utilized to isolate the fault and estimate the fault parameters (e.g. control effectiveness loss, stuck position, etc.). In the case of airframe fault, the system ID/extrapolation approach is utilized to identify the changes of system aerodynamic coefficients. The identified fault parameters will be applied to update onboard aircraft model based on which the flight envelope can be estimated. The flight envelope concept consists of an immediate flight envelope that is used by real-time envelope protection and adaptive flight control, and an extended flight envelope that is used by the Adaptive Trajectory Generator. To reduce the computational burden onboard the aircraft, the flight envelope for known fault scenarios (especially for actuator faults) can be evaluated offline. Therefore, a flight envelope database that was created offline can than be utilized by the online EEP system for envelope estimation via interpolation provided that the fault

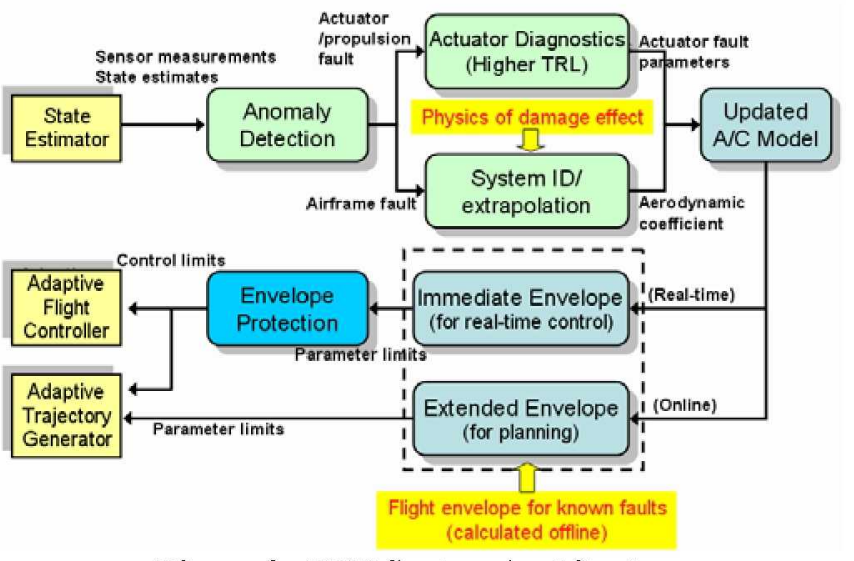

Figure 2. EEP System Architecture condition can be estimated accurately in real-time.

The extended flight envelope refers to the conventional flight envelope which is defined as a range of airspeed, attitude and normal load factor within which the aircraft can operate safely. Onboard estimation of the conventional flight envelope might not be practical in real-time since it concerns structure integrity. The assessment of the conventional flight envelope usually requires wind tunnel and structure integrity tests. Therefore its assessment can not possibly be done using only aerodynamic models. The immediate envelope refers to an achievable state envelope that the aircraft can reach given bounded-energy and bounded-magnitude inputs within a finite time horizon in the presents of flight anomalies. It can be estimated onboard using aerodynamic models. The immediately envelope estimation problem is closely related to envelope protection problem, while the conventional flight envelope will be useful for higher level functional modules such as trajectory generation and path planning.

Several fault modes are considered in this study: 1). control surface fault and failure including loss of effectiveness and stuck actuator. In this case, the aerodynamic derivatives will change according to the type of control-surface failure. As shown in Figure 2, an actuator fault will be detected by the anomaly detector and isolated by the actuator diagnostic module using a bank of nonlinear fault isolation estimators. The estimated fault parameters (control effectiveness coefficients, stuck position, etc) will be used to update online aircraft model. 2) propulsion fault and failure including effectiveness loss or total loss of thrust; propulsion system fault can usually be treated as an actuator fault at the flight controls level. 3) airframe/structural fault; the aerodynamics of an aircraft changes when some of its parts are lost. For example, if the aircraft loses part of or the entire vertical fin, the directional stability, spiral performance, and rudder effectiveness will worsen. As shown in Figure 2, system ID based approach will be utilized for airframe fault. Sensor faults are not considered in the current study. Several enabling technologies are presented in the following sections.

\section{Nonlinear Adaptive Fault Detection and Isolation Estimators (FDIE) for Actuator Faults}

An actuator fault model can be represented by additive and/or multiplicative faults as follows:

$$
u_{j}^{f}=\theta_{k}^{j} u_{j}+u_{j 0}
$$


where $u_{j}$ and $u_{j}^{f}$ represent the $\mathrm{j}$-th normal and faulty control actions. $u_{j 0}$ denotes a constant offset when the respective actuator is jammed and/or $0 \leq \theta_{k}^{j} \leq 1$ denotes a gain degradation (control effectiveness) of the $\mathrm{j}$-th component. The $\theta_{k}^{j}$ and/or $u_{j 0}$ parameters are estimated in real-time by the actuator FDIE module. The parameter estimates will be used to update the online aircraft model (GTM), which will be utilized to update flight envelope estimation.

\section{A. Online Actuator Fault Isolation and Estimation}

Consider a generic nonlinear aircraft model described by:

$$
\begin{aligned}
& \dot{x}=A x+\gamma(x, u)+\eta(x, u, t)+\beta\left(t-T_{f}\right) \phi(x, u) \\
& y=C x+d(x, u, t)
\end{aligned}
$$

where $x, u$, and $y$ are the state vector, input vector, and output vector of the engine system, $(\mathrm{A}, \mathrm{C})$ is an observable matrix pair. The equation

$$
\begin{aligned}
\dot{x}_{N} & =A x_{N}+\gamma\left(x_{N}, u\right) \\
y_{N} & =C x_{N}
\end{aligned}
$$

represents the known nominal nonlinear aircraft dynamics derived based on engine dynamics, while the healthy system is described by

$$
\begin{aligned}
& \dot{x}_{H}=A x_{H}+\gamma\left(x_{H}, u\right)+\eta\left(x_{H}, u, t\right) \\
& y_{H}=C x_{H}
\end{aligned}
$$

The difference between the nominal model and the actual (healthy) system is due to the term $\eta$ and $\mathrm{d}$, which represent the modeling uncertainties in the state and output equations. The changes in the system dynamics as a result of an actuator fault are characterized by $\phi(x, u)$.

Based on the system model, the following Fault Detection Estimator (FDE) is chosen:

$$
\begin{aligned}
& \dot{\hat{x}}^{0}=A \hat{x}^{0}+L\left(y-\hat{y}^{0}\right)+\gamma(y, u), \quad \hat{x}^{0}(0)=0 \\
& \hat{y}^{0}=C \hat{x}^{0}
\end{aligned}
$$

where $\hat{x}^{0}$ and $\hat{y}^{0}$ denote the state and output vectors estimated from this FDE respectively, and L is a design gain matrix. Note that for an aircraft using full state feedback control, $\mathrm{C}$ is usually an identity matrix, therefore the term $\mathrm{y}$ is used in the $\gamma$ function. A fault is detected when at least one component of the modulus of the output estimation error $\varepsilon_{y_{j}}^{0}(t)$ exceeds its corresponding threshold $\bar{\varepsilon}_{y_{j}}^{0}(t)$.

The bank of fault isolation estimators (FIEs) is activated only after a fault is detected for the purpose of fault isolation. Each FIE is designed based on the functional structure of a particular actuator fault. Let us denote $\hat{y}^{s}(t)$ as the estimated output vector generated by the $s$-th FIE, where $s \in\{1, \cdots, l\}$. Adaptive thresholds for each FIE are designed in such a way that in the presence of a fault in the $s$-th actuator, the residuals generated by all FIEs may exceed their corresponding thresholds, except for the $s$-th FIE. Note that the adaptive thresholds are functions of states and inputs and threshold values change when the operating condition changes, thus eliminating the need of model linearization and blending as many other methods do ${ }^{15}$. Proof-of-concept studies for this method have been conducted on the GTM model and simulation results are shown in Section VII.

\section{Online System Identification and Progressive Model Updating}

An important consideration with online system ID-based approaches is that they typically only identify the system dynamics in the vicinity of the operating condition where identification takes place. Before the unknown full flight 
envelope has been visited (which might not be recommended for an impaired aircraft), the available system performance limit won't be completely known. Therefore, the flight envelope estimation process will have to be conducted as a progressive process in which the envelope estimation accuracy will be continuously improved as more operating conditions are visited safely.

\section{A. Online System Identification}

An accurate aerodynamic model estimate is required for determination of flight envelop estimation. Aerodynamic models are readily available for aircraft resulting from wind-tunnel analysis and can be updated to accurately describe aircraft motions offline. However, when failures are introduced such as damaged surfaces, wings, fuselage, etc existing aerodynamic models would not truly represent aircraft responses. Therefore, the aerodynamic models should be updated in real-time reflecting the failures based on aircraft measurement sensor information. We use a strategy where the existing aircraft wind tunnel model is used and updates to the existing model are identified. The nominal aerodynamic model is not disregarded but used as a basis for the updated model aiding the estimation process to converge on an accurate aerodynamic model more quickly and more precisely. As example of the methodology, consider the following aerodynamic model for coefficient of drag:

$$
\begin{aligned}
C_{D}= & C_{D_{r}}(\alpha, h, m, q, \dot{\alpha}, \delta e, \ldots)+\Delta C_{D_{0}}+\Delta C_{D_{V}} \Delta V+\Delta C_{D_{\alpha}} \Delta \alpha+\Delta C_{D_{\alpha^{2}}} \Delta \alpha^{2} \\
& +\Delta C_{D_{\delta_{e}}}|\Delta \delta e|+\Delta C_{D_{\delta e^{2}}} \Delta \delta e^{2}+\frac{\bar{c}}{2 V}\left(\Delta C_{D_{q}} \Delta q+\Delta C_{D_{\dot{\alpha}}} \Delta \dot{\alpha}\right)
\end{aligned}
$$

Where $C_{D_{T}}(\alpha, h, m, q, \dot{\alpha}, \delta e, \ldots)$ is the nominal aerodynamic model obtained from wind tunnel testing (usually a nonlinear table lookup model) and the terms following the $\Delta C$ symbol represent increments to the nominal model and are to be identified in real-time based on sensor information. The coefficient of drag can be estimated from sensor measurement using the following:

$$
n_{x_{z}}=\frac{-\bar{q} s C_{D} \cos \alpha+\bar{q} s C_{L} \sin \alpha+T_{X}}{m}+n_{x_{b}}
$$

Where $n_{x_{z}}$ is obtained from an accelerometer sensor, $n_{x_{b}}$ is determined by the sensor consistency models, $T_{X}$ is the thrust estimate. If the angle-of-attack is small then Eq. (7) is approximated by:

$$
n_{x_{z}} \approx \frac{-\bar{q} s C_{D}+T_{X}}{m}+n_{x_{b}}
$$

Therefore, Eq. (6) may be written as:

$$
\begin{aligned}
J & =-\frac{m}{\bar{q} S}\left[n_{x_{z}}-n_{x_{b}}\right]-C_{D_{T}}(\alpha, h, m, q, \dot{\alpha}, \delta e, \ldots)-\left[\Delta C_{D_{0}}+\Delta C_{D_{V}} \Delta V+\Delta C_{D_{\alpha}} \Delta \alpha+\Delta C_{D_{\alpha^{2}}} \Delta \alpha^{2}\right] \\
& \left.-\left[\Delta C_{D_{\delta_{e}}}|\Delta \delta e|+\Delta C_{D_{\delta_{e}}} \Delta \delta e^{2}+\frac{\bar{c}}{2 V}\left(\Delta C_{D_{q}} \Delta q+\Delta C_{D_{\dot{\alpha}}} \Delta \dot{\alpha}\right)\right]\right]
\end{aligned}
$$

A linear regression method now be used to estimate $\Delta C$ terms using Eq. (9) updating the aerodynamic model. The linear regression routine minimizes Eq. (9). The proposed method has several advantages:

1. A simplified linear regression scheme can be used to estimate the model increments in real-time

2. No numerical integration of dynamical equations are required

3. The nominal aerodynamic model is utilized as a starting point and basis for the updated model

4. The identified increments are non-dimensional and are used for estimating the flight envelope at other flight conditions.

A similar approach can be developed for estimating lift, side force, and moment coefficients. Preliminary simulation has been conducted on the GTM model using a regularized recursive least square algorithm ${ }^{16}$. 


\section{B. Progressive Aerodynamic Model Updating}

The difficulty with flight envelope determination is in estimation of an accurate valid aerodynamic model given limited measurement data encompassing all flight condition of interest. Updating an aerodynamic model in the presence of a failure at a given flight condition (e.g. for cruising, referred to as Condition 1) with given flight measurement data at the current flight condition can be performed in real-time as discussed in previous sections. However predicting the flight envelop at a different flight condition say for a landing approach (referred to as Condition 2) based on the update at Condition 1 with no measurement data currently available for Condition 2 is another difficult problem. This prediction is essentially a constrained extrapolation problem. Without getting into mathematically complication, we will illustrate the approach with a simple linear extrapolation using the pitching coefficient $(\mathrm{Cm})$.

Consider a nominal aerodynamic table lookup model for determining the nondimensional pitching coefficient of an aircraft due to changes in elevator deflection as a function of angle-of-attack and elevator deflection assuming no failure, i.e.:

$$
\left.C m_{\delta e}\right|_{0}=f(\alpha, \delta e)
$$

Eq. (10) represents a nonlinear table lookup function valid for the entire operating flight condition of the aircraft. However, if the elevator surface effectiveness is reduced by $25 \%$ updating this nonlinear function to adequately estimate the reduced surface effectiveness or any other type of failure is difficult and may not be possible. Also, the form, in general, is nonlinear requiring increased computational effort for computers. In our approach, an alternate form of the model was utilized to overcome these difficulties based on an increment to the nominal model:

$$
\left.C m_{\delta e}\right|_{f}=\left.C m_{\delta e}\right|_{0}+\left.\Delta C m_{\delta e}\right|_{1} \Delta \delta e
$$

In this model form the additional linear increment, $\left.\Delta C m_{\delta e}\right|_{1}$ is identified for updating the model in the presence of the failure for flight Condition 1. The new updated model should be valid within a neighboring vicinity of the flight condition used to update the model assuming the linear assumption holds true. However, this approach has two advantages: 1) the nominal nonlinear model information is not disregarded and used in developing a new updated model and; 2) the update is linear and more easily estimated using reduced computational effort. The identified increment is also used to estimate the flight envelop at a different condition (e.g., Condition 2) with no measurement data required for flight Condition 2. The increment found for Condition 1 is valid for Condition 2 since it is nondimensional. However, there are dynamic pressure difference effects between Condition 1 and Condition 2 that should be accounted. Therefore, the following linear update on the identified increment for predicting behavior at the new flight condition, i.e. Condition 2 can be utilized.

$$
\left.\Delta C m_{\delta e}\right|_{2}=\left.\frac{\bar{q}_{2}}{\bar{q}_{1}} \Delta C m_{\delta e}\right|_{1}
$$

where the new increment is scaled by the ratio of the trim dynamic pressures from flight Condition 1 to flight Condition 2. Other more sophisticated nonlinear extrapolation can certainly be applied to improve the prediction accuracy. For example, the basic form of the nominal curves (e.g. using polynomial) can be applied to the new model. However, it should be pointed out that the updated model has a higher accuracy in the vicinity of current and previously visited operating conditions and deviates from the true dynamics for an operating condition that differs significantly from what has been seen. Therefore, the whole flight envelope estimation process should be conducted as a progress process as illustrated in Figure 1.

\section{Flight Envelope Estimation}

\section{A. Flight Envelope Estimation for Actuator Faults}

Compared with airframe faults, the actuator faults are much easier to model and estimate. As shown in Figure 3, the online flight envelope estimation for flight control actuator faults can be conducted in two steps: first, the FDIE module detects the fault and estimates the fault severity level; then the flight envelopes from the offline flight envelope library will be retrieved and interpolated to provide an accurate estimation of the flight envelope. This 
approach has several advantages: 1) the flight envelopes for different fault scenarios in the offline flight envelope library can be made very accurate by conducting wind tunnel tests, thus accuracy is guaranteed as long as the online fault severity estimation is accurate; 2) the online flight envelope is generated by simply interpolating existing flight envelopes from the offline-generated flight envelope library, thus the approach is computationally efficient; 3 ) the implementation is straightforward. However, this interpolation approach can not easily be extended to cover airframe faults. It's also worth noting that updated aircraft model with estimated actuator fault parameters can also be utilized by the reachability-based approach that is presented in the following section to calculate flight envelope updates.

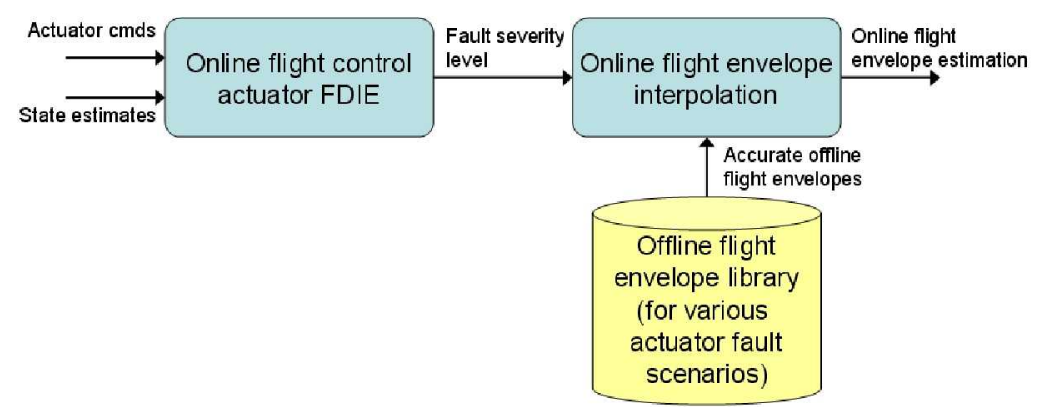

Figure 3. Online flight envelope estimation for actuator faults

\section{B. Flight Envelope Estimation Using Reachability Theory and Level Set Method}

Given dynamic system $\dot{x}=f(x, u)$, where $x \in R^{n}, u \in U \in R^{m}$. Let $\mathscr{Q}_{\left[t, t^{\prime}\right]}$ denote the set of Lebesgue measurable functions from the interval $\left[t, t^{\prime}\right]$ to $U$. Given the horizon $T \geq 0$ and a set of states $K \in R^{n}$, the following viability and reachability problems can be formulated ${ }^{6}$ :

- Viability: Does there exist a $u(\cdot) \in \mathscr{U}_{[t, T]}$ for which the trajectory $x(\cdot)$ satisfies $x(\cdot) \in K$ for all $t \in[0, T]$ ?

- Reachability: Does there exist a $u(\cdot) \in \mathscr{Q}_{[t, T]}$ and a $t \in[0, T]$ such that the trajectory satisfies $x(t) \in K$ ?

With this problem formulation, the flight envelope estimation is to characterize the set of initial states for which the answer to the viability/reachability questions is "yes", i.e. this set of initial states defines an envelope for the aircraft to be in if it intends to reach/remain in state set $\mathrm{K}$ in finite time.

After the system model is updated by the system ID and/or fault detection/isolation estimators, reachability-based methods can be utilized to calculate flight envelope in a progressive way. A conservative choice of target set $K$ not only helps to secure flight safety by applying conservative parameter limits, but also speeds up the reachable set calculation by restricting the optimization space. For example, when the aircraft detects a failure at a cruise speed of 400 knots at the altitude of 20,000 feet, the set of initial states for calculating the reachable set can be set to the range of altitude in between 15,000 to 20,500 feet and airspeed in between 350 to 450 knots. The parameter limits calculated from the reachable set will be used by the envelope protection module to prevent exceedance of flight envelope while the aircraft controller brings the aircraft to approach landing conditions (if landing is an option). As the altitude of the aircraft decreases, new set of initial states can be set and the calculation of reachable set be repeated in a progressive fashion.

\section{Intelligent Adaptive Flight Envelope Protection System}

This effort leverages the previous work carried out at Georgia Tech by a coauthor on reactionary envelope protection methodology ${ }^{11,17}$. The reactionary envelope protection method is similar in philosophy to obstacle avoidance. In obstacle avoidance, when an obstacle is detected in the path of a vehicle, a new safe trajectory is prescribed, and collision with the obstacle is avoided by following the safe trajectory. Similarly, the reactionary envelope protection method treats the limit boundary (upper or lower limit) as an 'internal' obstacle to the vehicle in its state space. This treatment allows the method to be applicable for both steady-state response critical as well as transient response critical limits. 
The reactionary envelope protection method relies on predicting future envelope violations using finite-time horizon prediction of the limit parameter response, and hence, requires an accurate representation of the limit parameter dynamics over the prediction horizon. In the absence of the availability of an accurate model of the limit parameter dynamics, an approximate model augmented with adaptive neural nets is used. A single hidden layer neural network with sufficient number of neurons and appropriate interconnection weights can approximate a continuous function in a compact domain to a desired level of accuracy $^{18}$. A block diagram representation of the adaptive estimation architecture is shown in Figure 4. As the linear model included in Figure 4 will be a poor global approximation of the limit parameter dynamics, it is augmented with an adaptive neural network block to obtain an improved local estimate of the limit parameter dynamics. Also, it includes an additional error feedback terms which improves

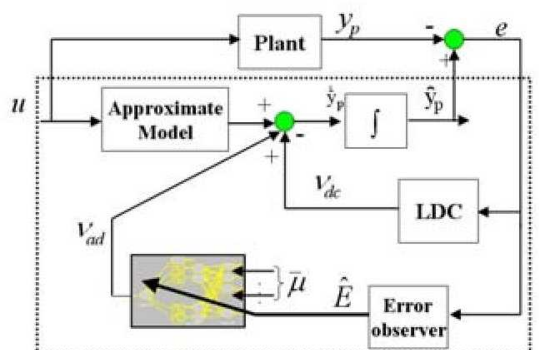

Figure 4. Adaptive Estimation of Limit Parameter Dynamics model convergence.

The adaptive estimate is used for predicting limit parameter response, and hence, any envelope violation in the future (over a selected horizon) to current control/command inputs. If a future violation is predicted, appropriate command/control corrections are then provided to enable the limit parameter response to effectively track a safelimit-response profile. The safe-response-profile of the limit parameter is constructed (Figure 5) by assuming the existence of an imaginary circular obstacle and the direction of the safe-response-profile is chosen to be along the tangential path in avoiding collision with the imaginary obstacle.
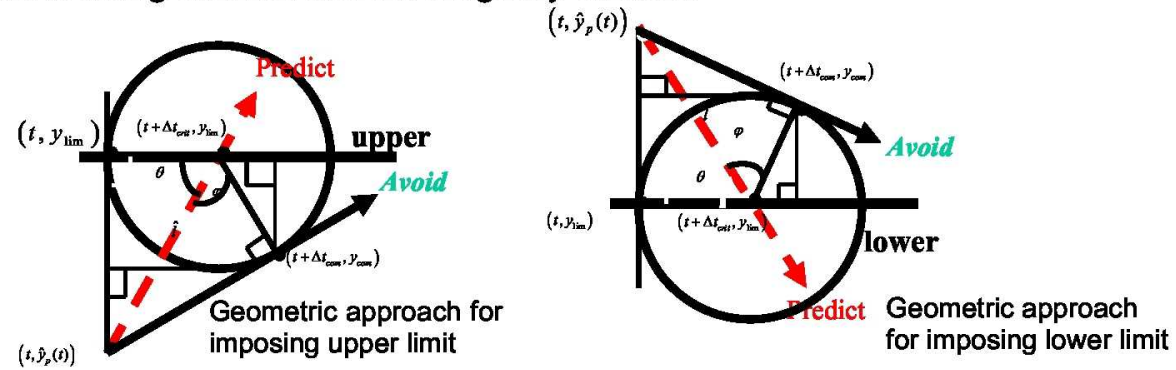

Figure 5: Safe-response-profile for envelope protection near limit boundary.

The command/control corrections are computed based upon tracking the safe-response-profile. Using a model inversion approach, one such control/command correction is given by

$$
\left.u_{c o r}=\left[y_{s}^{r}-\left(\sum_{i=0}^{r-1} a_{i} y_{s}^{i}+\frac{\partial \hat{h}}{\partial u} u+v_{a d}(\bar{\mu})-v_{d c}\right)-K\left(\hat{y}_{p}-y_{s}\right)\right]_{]}\right]\left(\frac{\partial \hat{h}}{\partial u}\right)^{-1}
$$

where $y_{s}$ is safe-response profile, $r$ is relative degree of the limit parameter response, $\frac{\partial \hat{h}}{\partial u}$ is estimate of control sensitivity, $\hat{\mathrm{y}}_{\mathrm{p}}$ is estimate of limit parameter, $a_{i}, i=0,1,2, \ldots, r-1$ are linear parameters of the approximate dynamics of limit parameters, $v_{a d}$ is output of the neural network compensating for model uncertainty, $v_{d c}$ is output of the linear dynamic compensator for stabilizing linearized error dynamics, $\bar{\mu}$ is normalized input vector of the neural network, and $K$ is parameter of the linear dynamic compensator.

\section{Simulation Results}

The NASA General Transport Model (GTM) ${ }^{19,20,21}$ Matlab/Simulink simulation environment was utilized for initial proof-of-concept demonstration of the developed methods. The GTM is equipped with a micro-INS, which provided 3-axis linear accelerometer measurements, angular rate measurements, estimated attitude angles, and GPS velocity and position. Air data probes on each windtip measured angle of attack, sideslip angle, static pressure, and dynamic pressure. Measurements from static pressure sensors and ambient temperature sensors were used to compute air density and altitude. Engine speed in rpm was measured and used as input to an engine model to compute thrust. The engine model was identified from ground test data, with adjustments for ram drag identified 
from flight data. Potentiometers on the rotation axes of all control surfaces measured control surface deflections. Mass properties were computed based on measured fuel flow, pre-flight weight and balance, and careful inertia measurements of the aircraft on the ground. The pilot stick and rudder commands and throttle position were also measured and recorded. Flight data was collected at $50 \mathrm{~Hz}$. In the above formula, $\mathrm{m}$ is known for GTM. $a_{x}, a_{z}, \mathrm{~T}_{\mathrm{x},}, \mathrm{T}_{\mathrm{z}}, \mathrm{V}$, and $\alpha$ are measured or estimated by the Kalman filter. Therefore, in the software simulation environment, we assume the above measurements available.

\section{A. Online Flight Control Actuator FDIE on the GTM}

As a proof-of-concept demonstration, five fault isolation estimators were implemented for rudder, left and right ailerons, left and right elevators respectively on the GTM Simulink model. The inputs to the FDIE module are the actuator commands (from the output of the flight controller) and aircraft states (from onboard state estimator). The fault mode reported in this paper is control effectiveness loss. However, our simulation studied has shown that the approach worked with stuck actuator fault mode as well. The following FDIE logic is utilized.

Fault detection decision scheme: The decision on the occurrence of a fault (detection) is made when at least one component of the residual generated by the FDE exceeds its corresponding adaptive threshold.

Actuator fault isolation decision scheme: Adaptive thresholds for each FIE are designed, such that, in the event that a particular actuator fault occurs, all the components of the residual generated by the FIE using the correct fault functional structure will remain below their corresponding adaptive thresholds. The decision of the occurrence of a particular actuator fault (isolation) is made when at least one of the components of the residual generated by each of the remaining FIEs exceeds its corresponding adaptive threshold.

\section{Fault scenario 1: Left elevator 60\% control effectiveness loss, fault time $=10 \mathrm{~s}$}

Proper control excitation is needed for the detection and isolation of actuator fault. For this simulation scenario, the actuator control signals and responding system states are shown in Figure 6. The residual signals from the fault detection estimator (FDE) and the adaptive thresholds are also shown in Figure 6. Clearly the second residual signal (for $\mathrm{q}-$ pitch rate) exceeded the threshold at time 10.4 seconds. The residual signals from the all FIEs are shown in Figure 7. It can be seen that all the residual signals (blue curves) from the left elevator FIE remained below the threshold signals (red curves) in Figure 7(b). The estimated fault parameter (control effectiveness coefficient) is shown in Figure 7(a). The estimated coefficient value is very close to the simulated value (0.4). The residual signals from other FIEs are shown in Figure 7 (c) to (f) respectively. It can be observed that at least one residual signal exceeded its corresponding threshold value. Therefore, the fault was also correctly isolated.

\section{Fault scenario 2: Right aileron 60\% control effectiveness loss, fault time $=10 \mathrm{~s}$}

Similar actuator controls and flight condition were applied to this simulation scenario. To keep the paper brief, only the fault parameter estimation (Figure 8 right) and the residuals from the right aileron FIE (Figure 8 left) are shown. The fault parameter (control effectiveness coefficient) estimation is very close to the truth value (0.4). All the residual signals in Figure 8 (middle) remain below thresholds, while at least one residual signal exceeded its threshold in other FIEs.

Fault scenario 3: Rudder 60\% control effectiveness loss, fault time $=5 \mathrm{~s}$

Similarly, simulation results for a rudder fault are shown in Figure 9. The results for left aileron and right elevator are similar but are not included in this paper for brevity.
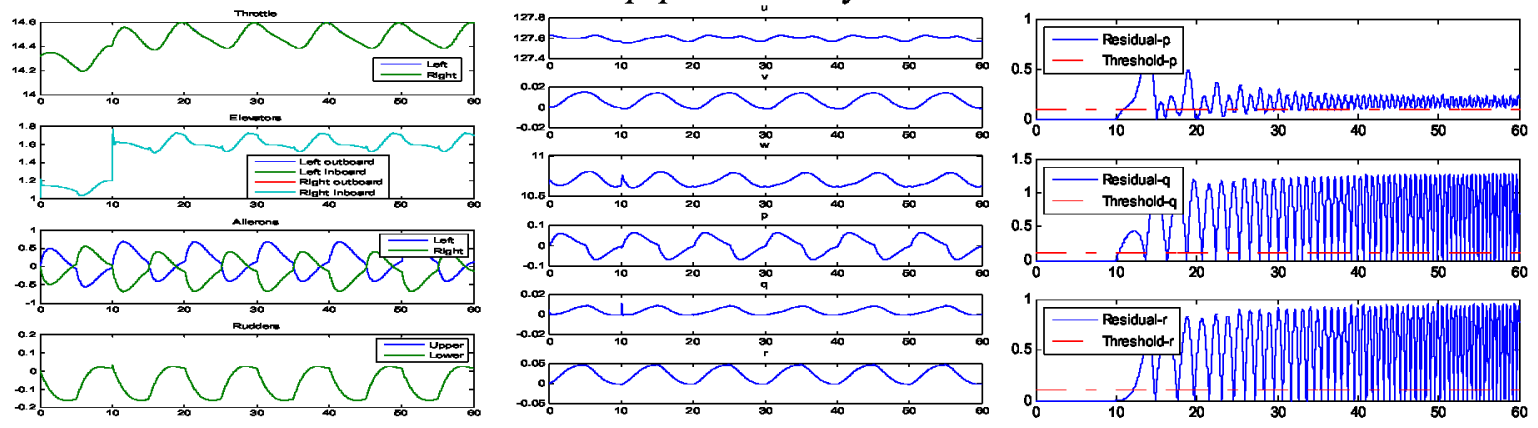

Figure 6: Actuator commands, system states, residual signals and adaptive thresholds from the FDE

(left: actuator commands from top: throttle, elevator, aileron, and rudder; middle: states from top: $\mathrm{u}, \mathrm{v}, \mathrm{w}, \mathrm{p}, \mathrm{q}, \mathrm{r}$; right plot: $\mathrm{p} / \mathrm{q} / \mathrm{r}$ residual signals (blue) and thresholds (red)) 


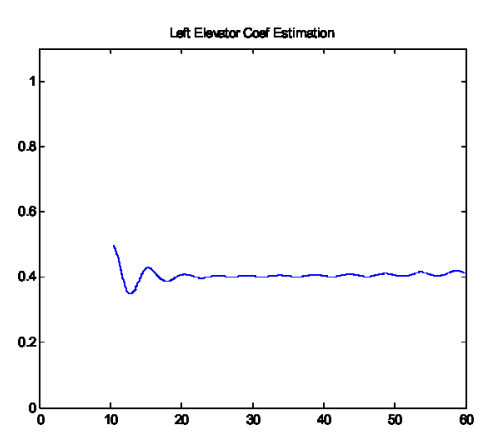

(a) control effectives estimate
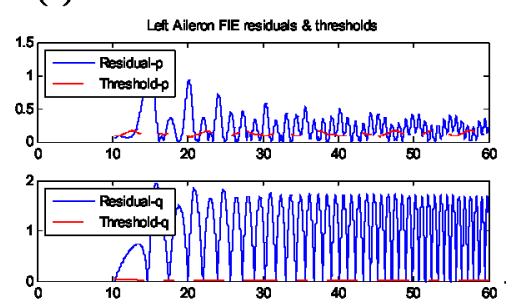

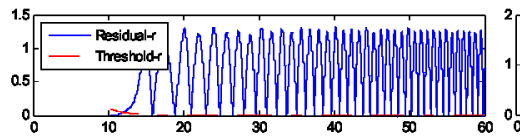

(d) left aileron FIE outputs

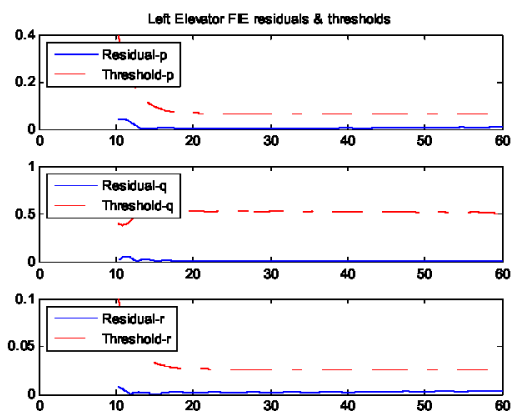

(b) left elevator FIE outputs
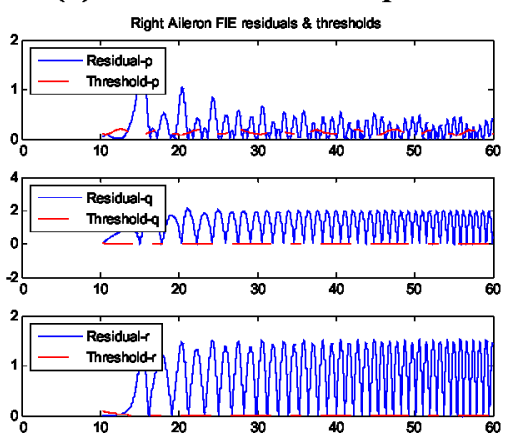

(e) right aileron FIE outputs

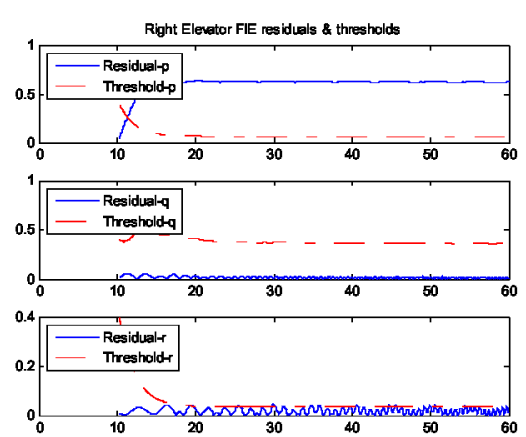

(b) right elevator FIE outputs
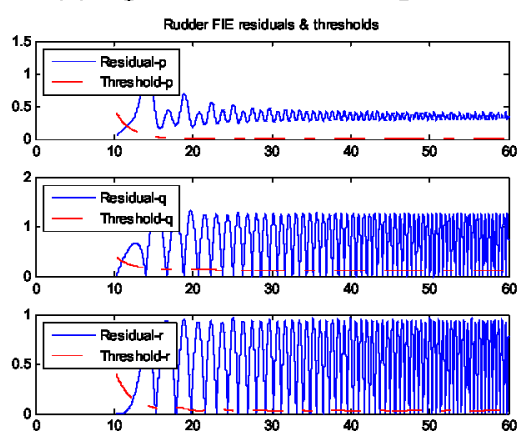

(f) rudder FIE outputs

Figure 7: Left elevator fault isolation and estimation results

(a: estimate of left elevator control effectiveness coefficient; $b$-f: the outputs from all five FIEs where the residual signals are blue and adaptive thresholds are red.)
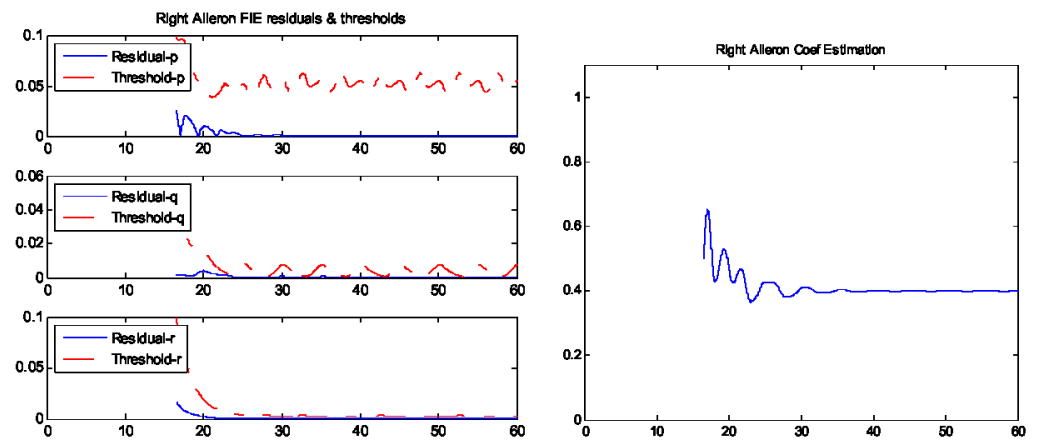

Figure 8: Right aileron fault isolation and estimation results

(left: the outputs from the right aileron FIE where the residual signals are blue and adaptive thresholds are red; right: estimate of right aileron control effectiveness coefficient;)
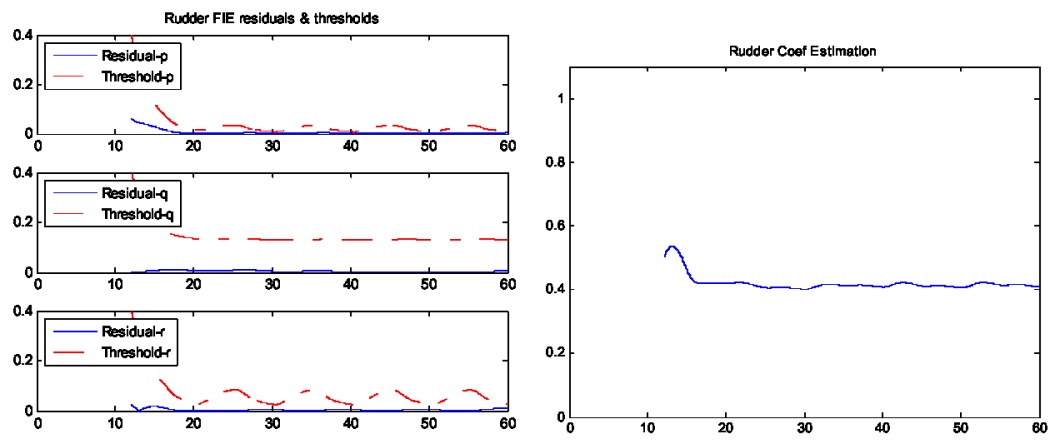

Figure 9: Rudder fault isolation and estimation results

(left: the outputs from the rudder FIE where the residual signals are blue and adaptive thresholds are red; right: estimate of rudder control effectiveness coefficient;) 


\section{B. Online System Identification and Reachability-based Flight Envelope Calculation}

A regularized recursive least square algorithm was utilized for online system identification of the changes of aerodynamic coefficients. As an improvement for fast detection and tracking in real-time, a change detection scheme and a time varying forgetting factor ahs been used. When a change in prediction residuals is detected, a smaller forgetting factor is utilized to discount 'old' data and place more weight on the latest measurements ${ }^{16}$. In this preliminary study, we assume the drag coefficient $\left(C_{D}\right)$ as a second-order polynomial function of angle of attack, i.e. $C_{D}=B_{0}+B_{1} \alpha+B_{2} \alpha^{2}$.

Some preliminary simulation results are shown in Figure 10. The aircraft is cruising at $130 \mathrm{ft} / \mathrm{s}$ at the altitude of 8000 feet when an alpha doublet input was used as the excitation signal for system ID. Figure 10 (a) shows the angle of attack response; Figure 10 (b) shows the drag coefficient, $C_{D}$, the identified $B_{0}, B_{1}, B_{2}$, and the identification error, respectively. Figure 10 (c) depicts the $C_{D}$ curve as a nonlinear function of angle of attack as described by the above polynomial function.

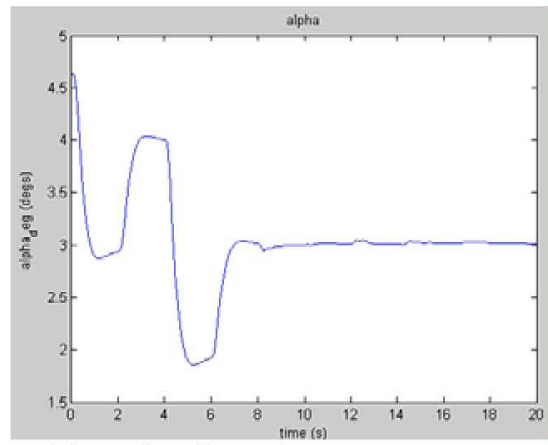

(a) angle of attack response

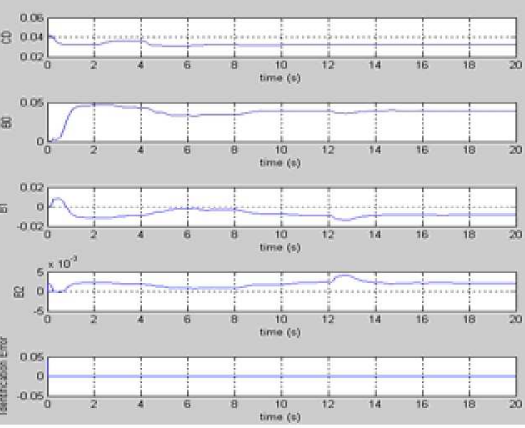

(b) identified coefficients

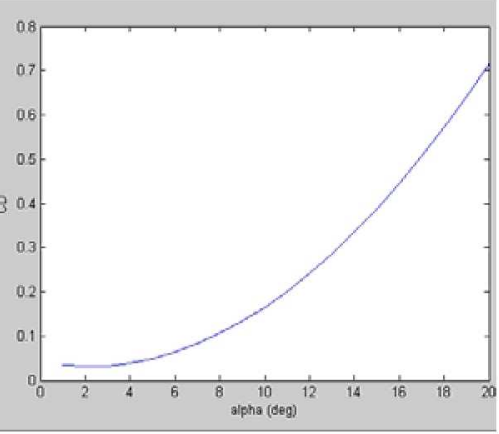

(c) $C_{D}$ as a function of $\alpha$

Figure 10: Online Identification of Aerodynamic Coefficients

The aerodynamic envelope for GTM fast dynamics is then calculated using the reachability approach. In the simulation, the following GTM and environmental parameters are utilized: $\rho=0.0023769$ Slugs $/ \mathrm{ft}^{3} ; S=5.9018 \mathrm{ft}^{2}$; $m=1.5416$ slugs; $\mathrm{T}_{\min }=0 ; \mathrm{T}_{\max }=50 \mathrm{lbf}$ (thrust). The baseline controller with angle of attack doublet as input is used for closed-loop GTM control. A Level Set Matlab Toolbox ${ }^{7}$ was be used for the numerical solution of the partial differential equation required to solve the problem. The initial rectangular envelope is selected as $50 \leq \mathrm{V} \leq 250$, $15^{\circ} \leq \gamma \leq 25^{\circ}, 0 \leq \mathrm{h} \leq 1500$, which are the initial bounds on airspeed, flight path angle and altitude. Figure 11 shows the flight envelope via reachable set calculation. Assuming the elevator surface gets stuck at 5 degree, Figure 12 shows the reduced flight envelope via the level set method. Comparing to Figure 11, the constraints on the responsiveness of elevator have resulted in noticeable reduction of the GTM achievable flight envelope.

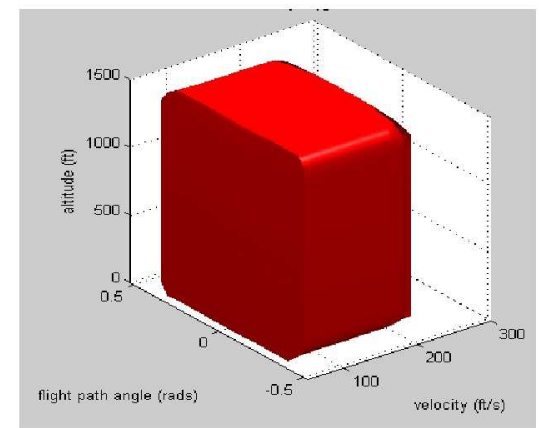

Figure 11: Calculated Flight Envelope for the nominal Case

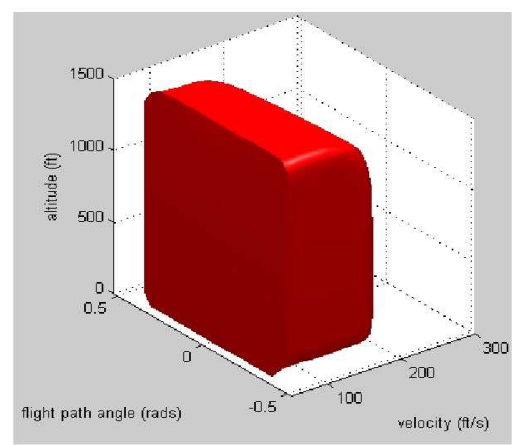

Figure 12: Reduced Flight Envelope with Stuck Elevator Actuators

The general shape of the level sets suggests that certain states are unsafe and should be avoided. If the aircraft ever gets to such a state, then the aircraft will soon violate the flight envelope requirements, therefore appropriate control actions are required to keep the aircraft in the safe envelope. If the initial condition is inside the level set, unsafe states can be avoided by applying the optimal controls whenever the state trajectory hits the boundary of the level set, hence keeping the aircraft in the desired safety envelope. 


\section{Progressive Model Updating with Linear Extrapolation}

The extrapolation method presented in Section IV was applied to the updating of the $\mathrm{Cm}$ (pitching moment) coefficient in the presence of an elevator actuator fault. Figure 13 displays the time history overlay data from the updated model comparing with the response from a faulted system with a simulated $25 \%$ reduction in elevator effectiveness for two flight conditions. The changes of aerodynamic coefficient (in this case, the pitching coefficient) were identified at flight condition 1 (cruise flight). The linear extrapolation method (Eq. 12) was utilized to predict the changes at flight condition 2 (landing). The updated model demonstrated outstanding agreement indicating a good prediction in the flight envelop. Note that the estimates of the aerodynamic coefficients will be updated/corrected as the aircraft approaches that particular operating condition, thus the model is updated progressively.

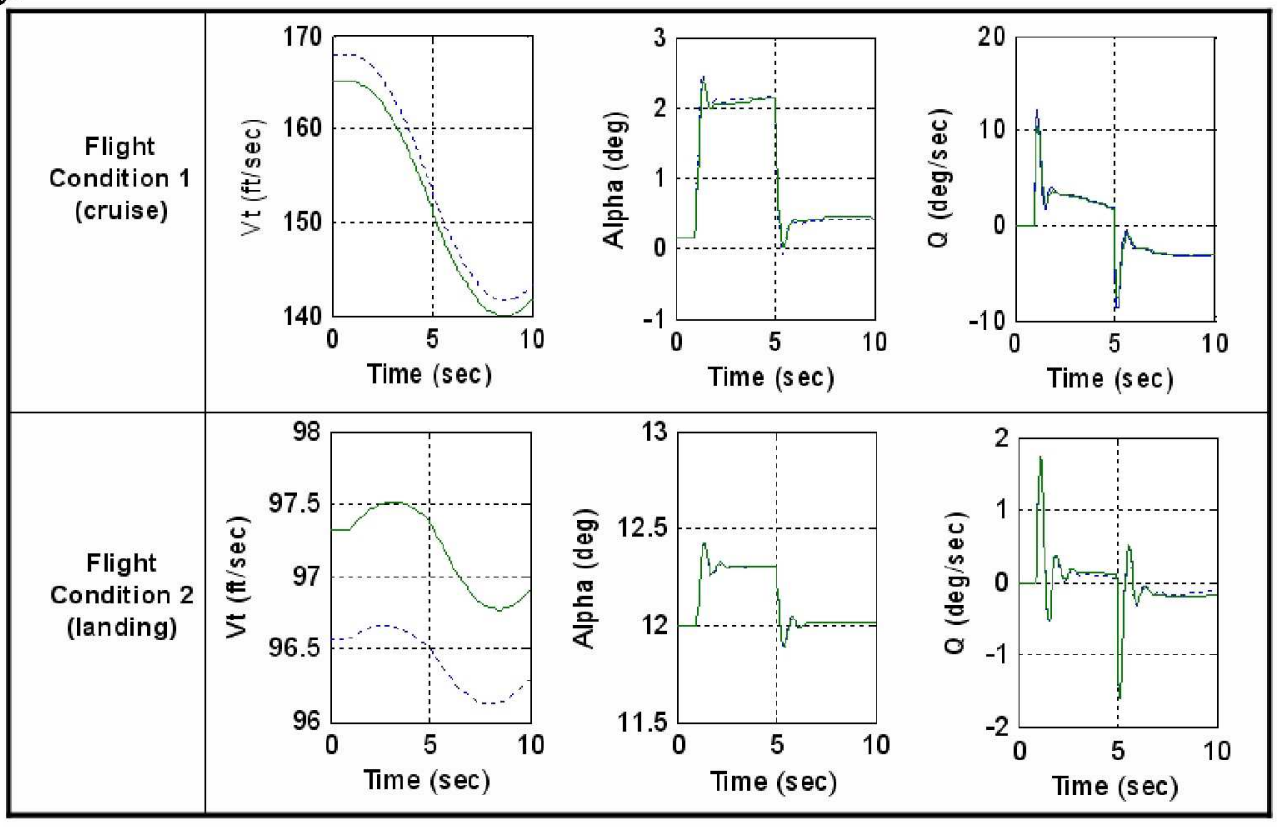

Figure 13: Performance of Updated Aerodynamic Model at Different Operating Conditions

(solid green: faulty system; dashed blue: updated model. Note that the aerodynamic coefficient changes were identified at flight condition 1 and extrapolated to flight condition 2)

\section{Intelligent Adaptive Flight Envelope Protection}

Simulation results for several different operating conditions and maneuvering scenarios have been produced. A representative result is shown in Figure 14 for the purpose of illustration.

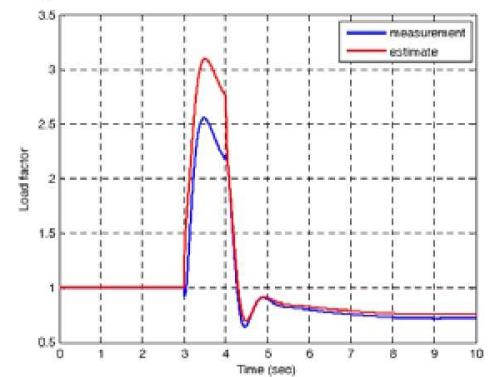

(a) without envelope protection

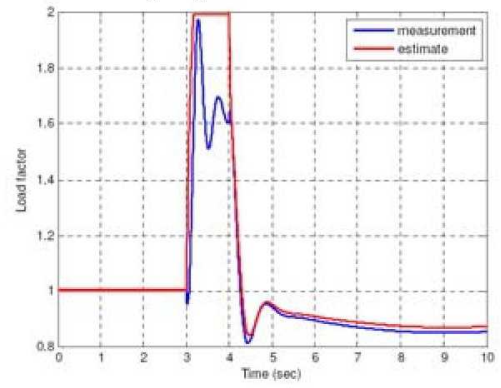

(b) Envelope protection with linear model

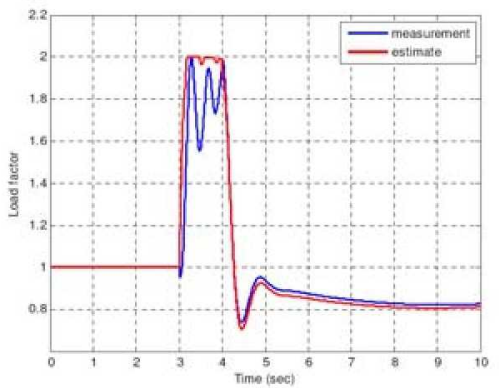

(c) envelope protection with NNenhanced model

Figure 14: Automatic Envelope Protection for GTM Load Factor

In this case, the aircraft was cruising at 90 knots. The simulated control input is an elevator pulse of -5 deg (to simulate a pull-up maneuver) at $t=3 \mathrm{sec}$ with a duration is $1 \mathrm{sec}$. A linearized model of load factor about the equilibrium point is used as an approximate model. The approximate model is corrected by including a linear correction term proportional to the difference between the true load factor value (measurement) and the estimated load factor value at each simulation step. The simulation is carried out at a fixed step size of $0.005 \mathrm{sec}$. The reactive 
limit parameter prediction horizon is taken to be 10 times the simulation step size, i.e., $0.05 \mathrm{sec}$ in the simulation results shown. Figure 14 (a) shows the simulation results without envelope protection. Assuming a limit of $2 \mathrm{~g}$ for load factor, the aircraft exceeded its load factor limit in this case. Figure 14 (b) shows the result of a linear model based envelope protection scheme, it managed to protect the aircraft against the limit, but the performance is compromised. Figure 14 (c) shows the result of a Neural Networks-enhanced envelope protection scheme, compared with the result in (b), the system not only protected the aircraft from exceeding load factor limit, but also pushed the performance to the allowed limitation.

\section{Conclusion and Future Work}

This paper reports the first phase of the development of an onboard adaptive flight envelope estimation and protection system. Several enabling techniques including real-time system identification and health assessment, progressive model updating, reachability-based flight envelope estimation and Neural-Networks based adaptive envelope protection, were presented. Simulation studies using the NASA GTM model have successfully demonstrated the feasibility of the presented concept and architecture.

Within the established architecture, future work will be focused on the following problems:

(1) Sensor consistence check; because the approach heavily relies on the quality of the measurement sensor systems, a novel sensor consistency check method is needed to provide a high degree of sensor measurement confidence ${ }^{22}$.

(2) Progressive model updating approach extended to cover all interested aerodynamic coefficients;

(3) Rigorous study on airframe faults with a focus on asymmetric aircraft model accounting for changes in aerodynamics, mass properties, wing area and the center of gravity.

(4) Expansion of the envelope protection algorithms to other limit parameters and the seamless integration of envelope estimation and protection modules.

\section{Acknowledgments}

This work is supported by the NASA under SBIR contract \# NNX09CE94P. The authors gratefully acknowledge the contributions from Jonathan DeCastro and Dr. George Vachtsevanos from Impact Technologies, LLC.

\section{References}

${ }^{1}$ Shah, G., Aerodynamic Effects and Modeling of Damage to Transport Aircraft, ALAA Guidance, Navigation and Control Conference and Exhibit, 18 - 21 Aug 2008, Honolulu, Hawaii.

${ }^{2}$ Bacon, B. J., and Gregory, I. M., General Equations of Motion for a Damaged Asymmetric Aircraft, AIAA Atmospheric Flight Mechanics Conference and Exhibit, 20 - 23 August 2007, Hilton Head, South Carolina.

${ }^{3}$ Nguyen, N., Krishnakumar, K., Kaneshige, J., and Nespeca, P., Flight Dynamics and Hybrid Adaptive Control of Damaged Aircraft, Journal of Guidance, Control, and Dynamics, Vol. 31, No. 3, Pp 751-764, May-June 2008.

${ }^{4}$ Murch, A. M., and Foster, J. V., Recent NASA Research on Aerodynamic Modeling of Post-Stall and Spin Dynamics of Large Transport Airplanes, 45th AIAA Aerospace Sciences Meeting and Exhibit, 8 - 11 Jan 2007, Reno, Nevada.

${ }^{5}$ Goman, M. G., Khramtsovsky, A. V., and Kolesnikov, E. N., Evaluation of Aircraft Performance and Maneuverability by Computation of Attainable Equilibrium Sets, Journal of Guidance, Control, and Dynamics, Vol. 31, No. 2, Pp. 329-339, MarchApril 2008.

${ }^{6}$ Lygeros, J., On reachability and minimum cost optimal control, Automatica, Volume 40, Issue 6, Pages 917-927, June 2004.

${ }^{7}$ Mitchell, I. M., The flexible, extensible and efficient toolbox of level set methods, J. of Scientific Computing, vol. 35, no. 23, pp. 300-329, 2008.

${ }^{8}$ Urnes, J. M., Reichenbach, Sr. E. Y., and Smith, T. A., Dynamic Flight Envelope Assessment and Prediction, $A I A A$ Guidance Navigation and Control Conference, 18-21 August 2008, Honolulu, Hawaii.

${ }^{9}$ Shin, J. and Belcastro, C., Robustness Analysis and Reliable Flight Regime Estimation of an Integrated Resilent Control System for A Transport Aircraft, AIAA Guidance Navigation and Control Conference, 18-21 August 2008, Honolulu, Hawaii.

10 Lombaerts, T. J. J., Huisman, H. O. , Chu, Q. P., Mulder, J. A. , and Joosten, D. A., Nonlinear Reconfiguring Flight Control Based on Online Physical Model Identification, Journal of Guidance, Control, and Dynamics, Vol. 32, No. 3, Pp 727748, May-June 2009.

11 Yavrucuk, I., Prasad, J. V. R., and Unnikrishnan, S., Envelope Protection for Autonomous Unmanned Aerial Vehicles, Journal of Guidance, Control, and Dynamics, Vol. 32, No. 1, Pp 248-261, January-February 2009.

12 Tang, Y., Atkins, E. M., and Sanner, R. M., Emergency Flight Planning for a Generalized Transport Aircraft with Left Wing Damage, AIAA Guidance, Navigation and Control Conference and Exhibit, 20 - 23 August 2007, Hilton Head, South Carolina. 
13 Byington, C.S., Watson, M., Edwards, D. and Stoelting, P., A model-based approach to prognostics and health management for flight control actuators, IEEE Aerospace Conference, Big Sky, Montana, 6-13 March 2004.

14 Balaban, E., Saxena, A., Bansal, P., Goebel, K.F., Stoelting, P., “A Diagnostic Approach for Electro-Mechanical Actuators in Aerospace Systems," IEEE Sensors Journal (accepted for publication)

15 Zhang, X., Polycarpou, M. M., and Parisini, T., Design and analysis of a fault isolation scheme for a class of uncertain nonlinear systems, IFAC Annual Reviews in Control, Volume 32, Issue 1, pp. 107-121, 2008.

16 Tang, L., Roemer, M., Bharadwaj, S., and Belcastro, C., An Integrated Aircraft Health Assessment and Fault Contingency Management System, AIAA Guidance, Navigation and Control Conference and Exhibit, Honolulu, Hawaii, 18 - 21 Aug 2008.

17 Unnikrishnan, S., Prasad, J.V.R. and Yavrucuk, I., Flight Evaluation of Reactionary Envelope Protection System for UAVs, Accepted for publication in the Journal of the American Helicopter Society, 2009.

18 Funahashi, K, On the Approximate Realization of a Continuous Mappings by Neural Nets, Neural Networks, vol. 2, issue 3, (1989) pp. 183-192.

19 Morelli, E. A., and Smith, M. S., Real-time dynamic modeling - data information requirements and flight test results, AIAA Atmospheric Flight Mechanics Conference and Exhibit, 18-21 August 2008, Honolulu, Hawaii.

20 Bailey, R. M., Hostetler, R. W., Barnes, K. N., Belcastro, C. M., and Belcastro, C. M., Experimental Validation: Subscale Aircraft Ground Facilities and Integrated Test Capability, AIAA Guidance, Navigation, and Control Conference, Washington, $\mathrm{DC}, 2005$

21 Murch, A. M., A flight control system architecture for the NASA AirSTAR flight test infrastructure, AIAA Guidance, Navigation, and Control Conference and Exhibit, 18-21 August 2008, Honolulu, Hawaii.

22 Crassidis, A. L., Identification Update of Aerodynamic Models with Sensor Consistency Checks, AIAA Atmospheric Flight Mechanics Conference and Exhibit, 16 - 19 August 2004, Providence, Rhode Island. 